\title{
Correlative 3D X-Ray, Laser Ablation, and SEM/EDS Mapping Establishing Access Point for FIB Tomography of Defects in Multi-Layer Ceramic Capacitors
}

\author{
J Favata $^{1}$, V Ray ${ }^{1}$ and S Shahbazmohamadi ${ }^{1}$ \\ ${ }^{1}$ REFINE Lab, University of Connecticut, 159 Discovery Dr. Storrs, CT, USA.
}

Multi-layer ceramic capacitors (MLCCs) are the most widely produced and utilized capacitors in all electronic equipment. It is estimated that one trillion $\left(10^{12}\right)$ pieces are incorporated to electronic systems every year [1]. The value of these components is underscored by their susceptibility to damage. Ceramic dielectric material is brittle and therefore susceptible to failure and cracking due to tensile and thermal stresses [2] to name a few. The traditional approach to root-cause failure analysis of manufacturing defects and operational failures of MLCCs relies on the lengthy and largely manual process of mechanical sectioning by either parallel lapping or cutting on a diamond saw, coupled with electrical testing and 2D imaging. Uncertainty of localization of mechanical cross-section, artifacts introduced by blade damage, sample contamination by blade materials or lapping media and cooling fluids, and shortcomings of 2D imaging all limit reliability and increase cost of mechanical sectioning. The destructive nature of mechanical deprocessing is an additional and significant risk factor affecting work with unique samples. We present a method leveraging correlative workflow of 3-D X-ray tomography, nanosecond pulsed laser milling, and FIB-SEM-EDS analysis which is conservative in its accessing of the defect site, threedimensional in nature, and coupled with in-situ monitoring of capacitor parameters.

Imaging and analysis were conducted on MLCCs with $\mathrm{BaTiO}_{3} \mathrm{~N}$ ceramic dielectric and Ni metallization which failed electrical testing. As the first step of the workflow, the sample was imaged by a Zeiss Xradia Versa 520 with a $150 \mathrm{kV} / 10 \mathrm{~W}$ beam at a voxel size of $1.3 \mu \mathrm{m}$ (Figure 1a). Upon examining the data, a suspect area was selected and targeted for a higher resolution X-ray tomography (Figure 1b) scan at a voxel size of $0.5 \mu \mathrm{m}$. Following localization of a suspected defect in three dimensions, the capacitor was mounted on a custom holder and the suspected defect was targeted with high-resolution laser machining and SEM/EDS imaging. X-ray tomography information was imported directly to a Zeiss Crossbeam 340 equipped with a Trumpf TruMark 6350 nanosecond pulsed laser. The data was registered within the Zeiss/Fibics Atlas 5 software package, cross-linking coordinate systems between the X-ray and FIB-SEMlaser-EDS systems and enabling correlative access to the defect location. Because the suspect area was considered too deep in all three directions for access by FIB milling, laser ablation was utilized for coarse deprocessing. The laser parameters $\left(20 \mathrm{~W}\right.$ average $/ \sim 33 \mathrm{~kW}$ peak power, $20 \mathrm{~ns}$ pulse width, and $300 \mathrm{~mm} \cdot \mathrm{s}^{-}$ ${ }^{1}$ raster speed) were optimized for highest rate of material removal with minimal melting, and multiple raster patterns were used to mitigate redeposition during the ablation process. First, the laser pattern was set to progress from the sample's edge inwards to the suspect area every $10 \mu \mathrm{m}$. Then, the laser was programmed to raster the same area at stepped angular offsets (Figure 1c). The process was repeated after rotating the sample $90^{\circ}$ for deprocessing in orthogonal direction. In-situ monitoring of resistance through capacitor was conducted with a 61/2-digit Keysight 34401A digital multimeter and a custom monitoring interface (Figure 2a) to observe effects of laser ablation on leakage in real time. Unique patterns of leakage variations were observed during lasering in different orientations, i.e. parallel vs. orthogonal to the capacitor's conductive plates (Figure 2b). After establishing the access point about 50 micrometers from the defect, SEM imaging and EDS mapping (Figure 3) were conducted on the targeted area to examine the extent of laser ablation quality and integrity of $\mathrm{BaTiO}_{3} \mathrm{~N}$-dielectric and $\mathrm{Ni}$-conductive layers. Initial inspection revealed re-deposition of ablated material as probable cause of reduced dielectric resistance. 
Additional step of "clean up" laser milling with low material removal rate allowed achieving "infinite" dielectric resistance, and thus zero measured leakage current. Depending on the defect dimensions gathered from X-ray CT and the level of through-dielectric current leakage present at the onset of the study, the lasering process can either: (1) continue progressing through the suspected defect region to collect sequential SEM/EDS data and learn if the suspect area removal prompts a drop in current leakage or (2) stop and be followed with FIB-SEM serial sectioning [3] and in-chamber electrical monitoring. The former could be preferable for large defects, while the latter could allow for more detailed information from the suspect defect area and improved correlation between the defect itself and the leakage current.

References:

[1] J Ho et al., IEEE Electrical Insulation Magazine. 26 (2010), p. 20.

[2] P O’Malley et al., 55 ${ }^{\text {th }}$ Annual NDIA Fuze Conference (2011), p. 269.

[3] J Favata et al., Microscopy \& Microanalysis 24 (2018), p. 362.
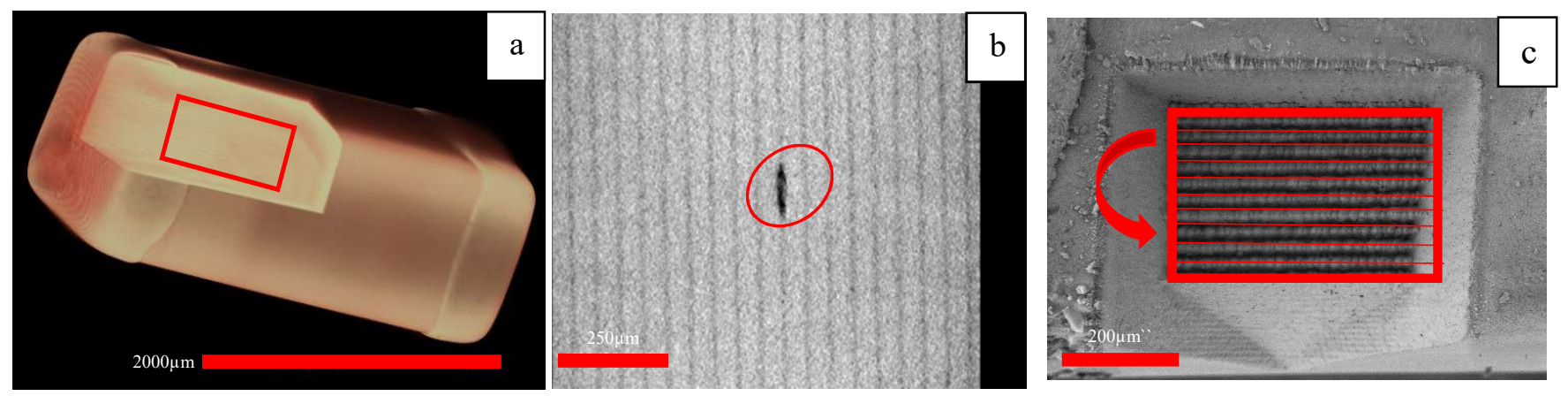

Figure 1. 3-D X-ray tomography volume rendering of MLCC (a), high-resolution cross-section of suspected defect area (b), and laser raster pattern with direction of incremental line rotation (c).
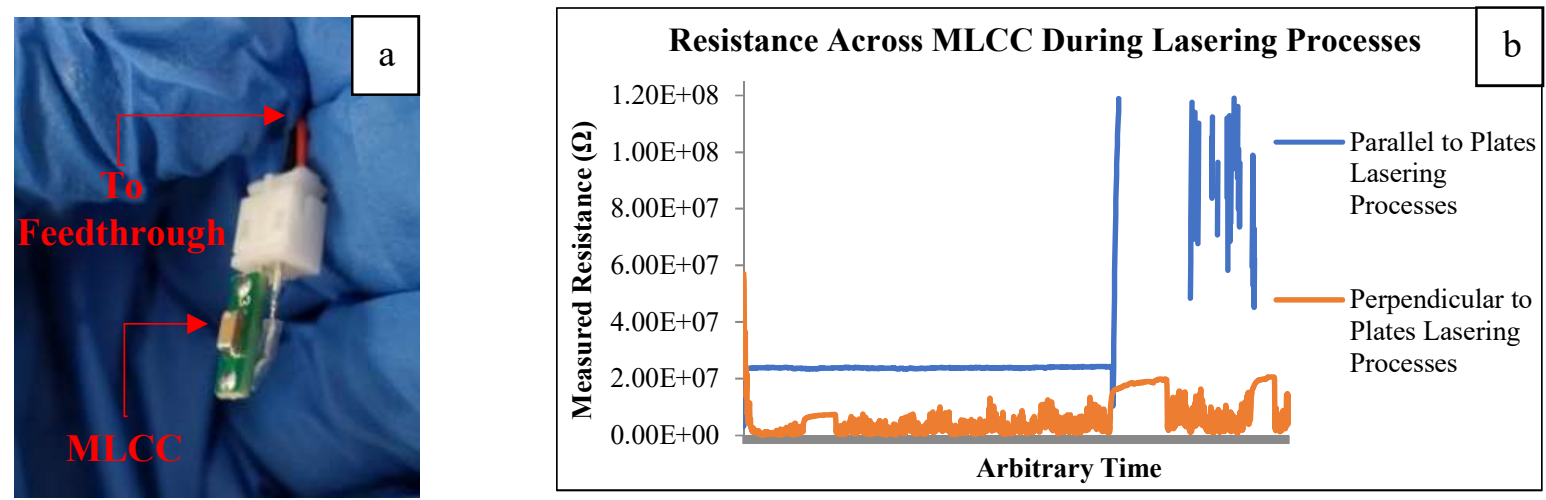

Figure 2. Sample-end in-situ monitoring configuration (left); Resistance measurements across MLCC during lasering processes; Breaks in blue graph represent infinite resistance, corresponding to no detectable leakage (right).
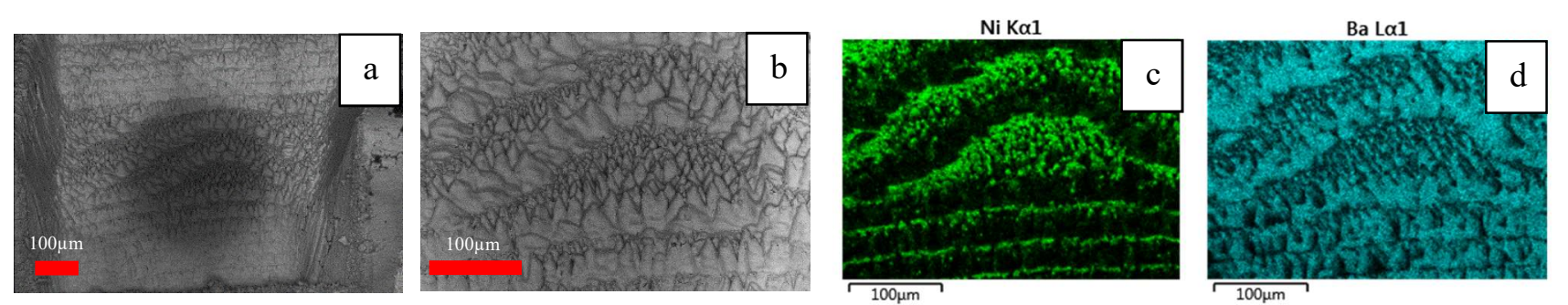

Figure 3. SEM images of access point to region of suspected defect (a and $b$ ) and respective EDS maps showing distinct $\mathrm{BaTiO}_{3}$-dielectric and Ni-conductive plate regions (c and d) after laser milling. 